\title{
MODELIZACIÓN ANALÓGICO-DIGITAL DE LA DIFUSIÓN DE CONTAMINANTES Y NUTRIENTES, EN CONDICIONES DE RÉGIMEN ESTACIONARIO EN EL EMBALSE FRONTAL DE TERMAS DE RÍO HONDO, REPÚBLICA ARGENTINA
}

\author{
Jorge G. Perera , Gustavo R. Sbrugnera , Oscar J. Graieb y Teresa C. Perera
}

Centro de Ingeniería Ambiental, Facultad Regional Tucumán, Universidad Tecnológica Nacional y Departamento de Posgrado, Facultad de Agronomía y Zootecnia, Universidad Nacional de Tucumán, San Martín 1327 - 4000 San Miguel de Tucumán, República Argentina - Tel. \& Fax: 543814213176

\begin{abstract}
RESUMEN
Se desarrolla una metodología de modelización de los procesos difusivos en condiciones de régimen estacionario, de contaminantes y nutrientes de origen superficial y puntual, aportados por los tributarios al Embalse Frontal de Termas de Río Hondo (Provincias de Tucumán y Santiago del Estero, República Argentina). El modelo analógico-digital, genera isolíneas de potencial V (curvas equipotenciales) o bien superficies reticuladas, que se corresponden con la dispersión espacial de contaminantes y nutrientes, en el medio líquido, cuando se emplea la Concentración C como parámetro distribuido. En los procesos de difusión y mezclado, en medio isótropo, con conductividad eléctrica $\sigma$, independiente de la posición r, es posible emplear un sistema análogo de conducción eléctrica continua óhmica descrito por la ecuación de Laplace $\left(\nabla^{2} \mathrm{~V}(\mathrm{r})=0\right)$. El modelo, electro-analógico bidimensional, fue construido a escala con papel semiconductor, respetando la planimetría del Embalse Frontal de Termas de Río Hondo, cuando el espejo alcanza la cota máxima. Se obtuvieron valores de tensión o potencial eléctrico y de este modo, se generaron soluciones para la ecuación de Laplace $\nabla^{2} \mathrm{C}(\mathrm{r})=0$, que describe la distribución espacial de la concentración $\mathrm{C}$ de contaminantes y nutrientes, con coeficiente de difusión o mezclado $\mathrm{D}$, independiente de la posición $\mathrm{r}$. El dispositivo experimental empleado, incluye además del modelo electro-analógico, un pantógrafo; un voltímetro; un conversor analógico-digital y dos programas computacionales para controlar las tensiones aplicadas en los electrodos, en función de los caudales másicos aportados por los tributarios y gestionar la adquisición de datos, para generar archivos de ternas de puntos, pertenecientes a las soluciones del problema.
\end{abstract}

Palabras clave: modelos analógico-digitales; difusión de Contaminantes y nutrientes; embalses; Ecuación de Laplace; régimen estacionario.

\begin{abstract}
A modelling methodology is developed of the diffusion process of contaminants and nutrients of superficial and precisely determined origin, in steady state conditions, in connection with the tributaries that contribute to the Termas de Rio Hondo Dam (Provinces ơ Tucumán and Santiago del Estero - Argentine Republic). The analogue-digital model generates potential isolines $V$ (equipotential curves) or reticulated surfaces that correspond to the spatial dispersion of contaminants and nutrients in $u$ liquid medium when the concentration $C$ is employed as a distributed parameter. It is possible to apply an analogue system of resistive DC electric conduction to diffusion processes, as described by the Luplace equation. Since the medium is isotropic with electric conductivity $\sigma$, independent of the position $r$, itfollows that: $\nabla^{2} V(r)=0$. The bidimensional electro-analogue model was constructed to scale with semi-conductor paper, respecting the bathymetry of the Termas de Rio Hondo Dam, when the level of the lake is at its maximum. Tension values were measured in order to generate solutions for the Laplace equation $\nabla^{2} C(r)=0$. This describes the spatial distribution of the contaminant and nutrient concentrations $C$ with a diffusion or mixing coefficient $D$, independent of position I: The experimental devices employed include, besides the electro-analogue model, a pantograph; a voltmeter; an analogue-digital converter and computer programs that control the voltages applied to the electrodes. The controlis done as afunction of the mass flow of tributaries and carry out the data acquisitionfor producing files with a group of three points each which belong the problem solution.
\end{abstract}

Keywords: digital-analogue models; diffusion of contaminants and nutrients; reservoirs; Laplace Equation; steady state.

Limnetica 20(I J: 8 I-Y2 (2001)

(.) Asociación Española de Limnología, Madrid. Spain. ISSN: 0213-8409 


\section{INTRODUCCIÓN Y ANTECEDENTES}

La elaboración y empleo de modelos analógicos, se basa en la circunstancia de ser descriptos dos sistemas físicos distintos por las mismas ecuaciones diferenciales generales, en las que difiere el significado físico de las variables (Liebmann, G., 1953). Consiguientemente, la solución de las ecuaciones diferenciales en un contexto, puede ser trasladada de otro, haciendo mediciones experimentales. Los problemas de campo se relacionan con parámetros distribuidos (distributedparameter), donde las entidades físicas consideradas, dependen de las coordenadas espaciales del sistema. Una o más variables independientes de posición, participan del problema, mientras que el tiempo es la única variable independiente en un modelo de parámetros concentrados o globales (lumped-parameter): se reconocen entonces, dos tipos diferentes de variables independientes, las espaciales y la temporal. En relación con las variables dependientes, se distinguen las llamadas across y las llamadas through; las primeras, relacionan la situación en un punto del campo con la correspondiente a otro punto en el mismo campo y como diferencia entre magnitudes, se expresan habitualmente en unidades relativas. En un sistema eléctrico, la variable across, es la tensión que puede medirse como diferencia entre el potencial eléctrico de dos puntos o bien como diferencia de potencial entre un punto del sistema y algún otro de referencia como tierra o potencial de tierra. En razón de ser medida como diferencia entre el potencial escalar de dos puntos, es una cantidad escalar. La variable through, representa el flujo de alguna cantidad que atraviesa una sección transversal elemental del campo. En un sistema eléctrico, through es el flujo de corriente; ésto es, la tasa de flujo de electrones por unidad de área. Esta cantidad es especificada mediante la medición hecha en un punto del campo, en términos de magnitud; dirección y sentido de flujo. Es consecuentemente una cantidad vectorial.

La relación entre las variables across y through se expresa en función de los parámetros del sistema. Estos parámetros para sistemas de campo o parámetros distribuidos y para sistemas de parámetros concentrados o globales, pueden dividirse en tres tipos diferentes conforme al rol que juegan en los mecanismos de transferencia, asociados a las características del sistema. Los modelos analógicos continuos que describen sistemas tridimensionales, deben ser tridimensionales. La capacitancia del análogo eléctrico de un sistema real, es en general baja, por ello, los procesos transitorios ocurren en tiempos cortos, de difícil medición experimental. Ésto se debe principalmente a que se pretende que el mismo material satisfaga requerimientos resistivos y capacitivos. En el caso de sistemas descriptos por la ecuación de onda amortiguada, los tres parámetros, disipatividad $D$; capacidad como reservorio de flujo por unidad de longitud del campo $E$, y capacidad como reservorio de potencial por unidad de longitud del campo $E_{\mathrm{p}}$, son importantes.

En un modelo de parámetros concentrados o globales (lumped-parameter), el sistema físico se considera compuesto por una red de diferentes elementos interconectados y se formula expresando las ecuaciones diferenciales parciales que lo describen, como diferencias finitas. Se consideran en general cinco tipos de modelos de parámetros concentrados o globales; éstos son, los sistemas traslacionales; los rotacionales; los eléctricos; los fluídicos y los térmicos (Avrami \& Paschkis, 1942). Para cada uno de ellos, se dispone de tres elementos básicos, tal como se ha expresado, correspondientes a la disipatividad; a la capacidad para actuar como reservorios de potencial y a la capacidad para actuar como reservorios de flujo por unidad de longitud del campo. Los tres elementos mencionados, en un circuito eléctrico son los resistores; los capacitores y los inductores.

Es objetivo principal del presente trabajo, desarrollar una metodología de modelización analógico-digital de los procesos difusivos en condiciones de régimen estacionario, de contaminantes y nutrientes de origen superficial y puntual, aportados por los tributarios al Embalse Frontal de Termas de Río Hondo. Este reservorio, originalmente de $1720 \mathrm{Hm}^{3}$, con un espejo de aproximadamente $34200 \mathrm{Ha}$, es el mayor 
embalse artificial de la Cuenca Salí-Dulce (Argentina). Se extiende desde el sureste de la Provincia de Tucumán, hacia la de Santiago del Estero, donde están emplazados el muro de cierre y la central hidroeléctrica. La problemática ambiental de la Cuenca Superior del Río SalíDulce, ha sido objeto de numerosos estudios por parte del Centro de Ingeniería Ambiental (CEDIA) de la Facultad Regional Tucumán, Universidad Tecnológica Nacional, Argentina (Perera et al., 1994).

\section{MATERIALES Y MÉTODOS}

En diversas ramas de la Ciencia y de la Tecnología se presenta el problema de resolver la ecuación de Laplace para determinadas condiciones de contorno; casos típicos son los del cálculo de campos eléctricos y magnéticos; de campos de velocidades en el movimiento de fluidos, tanto en el espacio libre como en medios porosos (Prasuhn, 1987); de distribuciones de temperaturas en transmisión del calor; de concentraciones en problemas de difusión y mezclado (Hoffmann et al., 1991; Hoffmann et al., 1993); etc.. A partir de sistemas de conducción eléctrica óhmica, donde la descripción del proceso está ligada a la ecuación de Laplace y pueden realizarse mediciones experimentales, se establecen analogías físico-matemáticas con sistemas reales.

En sistemas continuos, unidimensionales, con conductividad $\mathbf{O}(\mathrm{x}) \mathrm{y}$ aplicación de un potencial eléctrico $V(\mathrm{x})$, se tiene por unidad de área normal a la dirección $x$, una circulación de corriente de intensidad $J$, dada por:

$$
J=-\sigma(x) \frac{d V(x)}{d x} .
$$

Si se introduce un factor de proporcionalidad $\mathbf{x}$ en las ecuaciones de Laplace, se tiene: $\mathbf{O}(\mathbf{x}, y, z)$ $=a \mathrm{~V}(\mathrm{x}, y, z)$. La medición del potencial $V(x, y$, $z)$ en el sistema óhmico, proporciona una solución para un sistema con igual geometría, si los potenciales en el contorno se eligen de forma que: $\varnothing$ (en el punto de contorno $)=\mathrm{x} \quad V($ en el punto de contorno).
Si se consideran dos planos; uno en $x$ y otro en $\mathrm{x}$ $+d x$, la acumulación de carga entre ambos, estará dada por la diferencia entre la corriente que entra y la que sale, es decir:

$$
\begin{aligned}
\frac{\partial \rho}{\partial t} d x & =J(x)-J(x+d x)+f(x) d x= \\
& -\frac{\partial J}{\partial x} d x+f(x) d x
\end{aligned}
$$

donde $\rho(x, t)$ es la densidad de carga y $f(x)$ es la densidad de fuentes de carga. Utilizando la expresión que proporciona $J$ y eliminando el factor $d x$ se obtiene:

$$
\frac{\partial \rho}{\partial t}=\frac{\partial}{\partial x}\left(\sigma(x) \frac{d V(x)}{d x}\right)+f(x)
$$

Para el caso tridimensional se tienen tres términos similares al del segundo miembro; es decir:

$$
\begin{gathered}
\frac{\partial \rho}{\partial t}=\frac{\partial}{\partial x}\left(\sigma \frac{\partial V}{\partial x}\right)+\frac{\partial}{\partial y}\left(\sigma \frac{\partial V}{\partial y}\right)+ \\
\frac{\partial}{\partial z}\left(\sigma \frac{\partial V}{\partial z}\right)+f(x),
\end{gathered}
$$

utilizando las definiciones de gradiente y divergencia (Davis \& Snider, 1979), puede escribirse:

$$
\frac{\partial \rho}{d t}=\operatorname{div}(\mathbf{a} \operatorname{Grad} V)+f(x)
$$

Si la conductividad $\sigma$ no depende de la posición, se tiene:

$$
\frac{\partial \rho}{d t}=\sigma \nabla^{2} V+f(x)
$$

donde $\nabla^{2}$ es el operador laplaciano

$$
\nabla^{2}=\frac{\partial^{2}}{\partial x^{2}}+\frac{\partial^{2}}{a y^{2}}+\frac{\partial^{2}}{\partial z^{2}}
$$

Si la densidad de carga $\boldsymbol{p}$ no depende del tiempo (estado estacionario), en ausencia de fuentes $(f(x)=0)$, es: $\nabla^{2} V(x, y, z)=0$. 
Se aplican modelos electroanalógicos para simular la distribución espacial de contaminantes y de nutrientes, en las aguas de lagos y embalses. Este tipo de modelos es empleable tanto en el caso límite en que el mezclado de las aguas es dominante sobre la deriva, como en el que podría denominarse de flujo laminar. En el presente caso, se considera que la distribución de componentes está determinada por los procesos de mezclado. Indicando con $C(\mathbf{r})$ la concentración de un componente, función de la posición dada por el vector $\mathbf{r}$, el flujo $\mathbf{J}_{\mathrm{m}}$ del componente se expresa por: $\mathbf{J},=\mathbf{v} C-D$ Grad $C$, donde $\mathbf{v}$ es la velocidad de deriva de la masa líquida. Si $C$ (r) está determinada principalmente por los procesos de mezclado, el primer término del segundo miembro de la ecuación anterior, es despreciable, por lo que puede escribirse: $\mathbf{J},=-D$ Grad $C$. El coeficiente $D$ es el coeficiente de mezclado del sistema. En ausencia de fuentes y sumideros del componente en cuestión, por descomposición o por reacciones químicas; etc., la conservación de éste, implica que en régimen estacionario se tiene: $\operatorname{div} \mathbf{J}_{\mathbf{m}}=0$. Suponiendo que $D$ es independiente de la posición $\mathbf{r}$, resulta: $\nabla^{2} C(\mathbf{r})=0$. Análogamente, en un sistema isótropo con conductividad eléctrica $\mathrm{O}$; potencial eléctrico $\mathrm{V}(\mathbf{r})$, el flujo de corriente $\mathbf{J}$, , está dado por: $\mathbf{J}_{\mathbf{e}}=$ - $\mathbf{o} \mathbf{G r a d}$ $V$. Para el estado estacionario la conservación de cargas implica que: div $\mathbf{J},=0$.

Utilizando $\mathbf{J}_{\mathbf{e}}=$ - $\mathbf{o}$ Grad $V$, en sistemas homogéneos (oindependiente de la posición), se tiene: $\nabla^{2} V(\mathbf{r})=0$. La equivalencia matemática de esta ecuación con $\nabla^{2} C(\mathbf{r})=0$, puede emplearse para obtener soluciones, realizando mediciones del potencial eléctrico V (r) en un sistema de similar geometría. Esta analogía es de mérito práctico cuando los sistemas son bidimensionales; caso en que puede utilizarse una cuba electrolítica o una hoja de papel semiconductor, tal como el empleado en el modelo. Como la profundidad media del sistema real, es:

$$
\bar{z}=\frac{1720 \mathrm{Hm}^{3}}{34200 \mathrm{Hm}^{2}} \cong 0,05 \mathrm{Hm} \equiv 5 \mathrm{~m}
$$

y el diámetro equivalente, aproximadamente de 20867 m , se tiene una relación diámetro equiva- lente / profundidad media , superior a $4000: 1$. Esta circunstancia, respalda la simplificación conducente a un modelo bidimensional. De la comparación entre las ecuaciones $\nabla^{2} C(\mathbf{r})=0$ y $\nabla^{2} V(\mathbf{r})=0$, se infiere que el potencial eléctrico $\mathrm{V}(\mathbf{r})$ puede ponerse en correspondencia con la concentración $C(\mathbf{r})$ del componente de interés, que es en el caso de las corridas de simulación del presente trabajo, el ortofosfato soluble. Se elige este nutriente, en razón de su significación en el diagnóstico del estado trófico del embalse (Erickson, 1994; Glasson et al., 1994). Debe observarse que la mencionada correspondencia deja de ser válida en las proximidades de los puntos de ingreso de los tributarios en el lago o embalse, por la influencia de los caudales variables que aportan. El análisis de las condiciones de contorno en los puntos de ingreso de los tributarios, permite establecer los valores de caudales másicos correspondientes a los diversos potenciales eléctricos a aplicar en los electrodos que los emulan, en función de los correspondientes valores de caudal y concentración. Considerando los tributarios $1,2, \ldots, \mathrm{n}$; siendo $q_{\mathrm{i}}$ y $c_{\mathrm{i}}$ los respectivos caudales y concentraciones, con $\mathrm{i}=1,2, \ldots, \mathbf{n}$ y suponiendo que del lago o embalse sale una única corriente líquida, con caudal $q_{\mathrm{m}}$ y concentración $c_{\mathrm{m}}$ dados por: $q_{\mathrm{m}}=q_{1}+q_{2}+\ldots+q_{\mathrm{n}}-q_{\mathrm{e}}$, donde $q_{\mathrm{e}}$ representa la pérdidas por evaporación, se tiene que: $c_{\mathrm{m}} q_{\mathrm{m}}=c_{1} q_{1}+c_{2} q_{2}+\ldots+c_{\mathrm{n}} q$, Un tributario con caudal $q_{\mathrm{i}}$ y concentración $c_{\mathrm{i}}$, actuará como fuente o sumidero del componente, según sea $c_{\mathrm{i}}$ mayor o menor que $c_{\mathrm{m}}$, haciéndolo con una intensidad dada por: $q_{\mathrm{i}}\left(c_{\mathrm{i}}-c_{\mathrm{m}}\right)$.

Tomando una línea $L_{\mathrm{i}}$, alrededor de la desembocadura del tributario i , e indicando con $d L$ la longitud del elemento de línea y con $(\boldsymbol{G r a d} C)_{0}$, el gradiente de la concentración en la dirección ortogonal a $L$, el flujo del componente a través de la línea $L$ por efecto del mezclado, debe igualar a la intensidad de fuente (o sumidero) dada por $q_{\mathrm{i}}\left(c_{\mathrm{i}}-c_{\mathrm{m}}\right)$.

Se tiene entonces: $q_{\mathrm{i}}\left(c_{\mathrm{i}}-c_{\mathrm{m}}\right)=D I_{\mathrm{i}}, \mathrm{i}=1,2$, ..., $\mathrm{n}$, donde se ha empleado la notación: $I_{\mathrm{i}}=\int_{L}(\operatorname{Grad} C)_{0} d L$. Con la ecuación $c_{\mathrm{m}} q_{\mathrm{m}}=c_{1}$ $q_{1}+c_{2} q_{2}+\ldots+c_{\mathrm{n}} q_{\mathrm{n}}$ y las $\mathrm{n}$ ecuaciones: $q_{\mathrm{j}}$ (c, $\left.c_{\mathrm{m}}\right)=D I_{\mathrm{i}}$, se forma el siguiente sistema de $\mathrm{n}+$ 1 ecuaciones en $c_{1}, c_{2}, \ldots, c_{\mathrm{n}}, c_{\mathrm{m}}$ : 


$$
\left\{\begin{aligned}
q_{1} c_{1}+q_{2} c_{2}+\cdots+q_{n} c_{n}-q_{m} c_{m} & =0 \\
q_{1} c_{1}-q_{1} c_{m} & =D I_{1} \\
q_{2} c_{2}-q_{2} c_{m} & =D I_{2} \\
& \cdots \\
q_{n} c_{n}-q_{n} c_{m} & =D I_{n}
\end{aligned}\right.
$$

La columna de términos independientes es proporcional al coeficiente de mezclado $D$, por lo que todas las concentraciones, obtenidas del sistema de ecuaciones precedente, lo contendrán como factor. El valor absoluto de las concentraciones se obtiene dando el valor absoluto de una cualquiera de ellas, como por ejemplo $c_{\mathrm{m}}$, También pueden calcularse los caudales suponiendo que se conocen las concentraciones $c_{\mathrm{i}}$, (i $=1,2, \ldots, \mathrm{n})$ y $c_{\mathrm{m}}$. En efecto, de $q_{\mathrm{i}}\left(c_{\mathrm{j}}-c_{\mathrm{m}}\right)=D$ $I_{\mathrm{i}}, \mathrm{i}=1,2, \ldots, \mathrm{n}$, se obtiene:

$$
q_{i}=\frac{D I_{i}}{c_{i}-c_{m}}, i=1,2, \ldots, n .
$$

En síntesis, a los efectos de la rnodelización, sólo interesan los valores relativos de los caudales (Chow et al., 1994; Wanielista, 1990). La Tabla 1, presenta valores medios de los caudales hídricos; de las concentraciones y de los caudales masicos de fósforo soluble aportado por los tributarios del Embalse Frontal de Termas de Río Hondo.

El hardware empleado en la simulación consta de los siguientes cuatro componentes: modelo electro-analógico; pantógrafo; interfase analógico-digital (EDP) y computadora. El modelo electro-analógico, es una figura plana de papel semiconductor o resistivo, marca Teledeltos, cuyo contorno reproduce en escala $1: 150000$, la superficie del agua o espejo del lago, en la cota de $275 \mathrm{~m} \mathrm{snm}$. Se ha montado sobre un soporte piano dieléctrico. En los puntos de ingreso de cada tributario se ha instalado un electrodo de aluminio, el que conectado a una fuente de tensión regulable, permite simular el aporte de la substancia en estudio. En correspondencia con la descarga del embalse, también se ha instalado un electrodo de similares características, tal como ilustra el Plano del Modelo Electro-analógico (Fig. 1). La magnitud eléctrica considerada es la resistencia. El embalse es representado por una malla completa de resistencias entre todos los electrodos conectados, como se muestra en el Circuito Eléctrico Equivalente del Modelo Analógico (Fig. 2).

\begin{tabular}{|l|lrrrr}
\hline $\begin{array}{l}\text { Caudales hídricos } \\
\text { aproximados } \\
\text { en } \mathrm{m}^{3} \mathrm{~s}^{-1}\end{array}$ & $\begin{array}{l}\text { Semestre húmedo (noviembre a abril) } \\
\text { Primer trimestre seco (mayo a julio) }\end{array}$ & 81.0 & 31.0 & 35.0 & 147.0 \\
& $\begin{array}{l}\text { Segundo trimestre seco o crítico } \\
\text { (agosto a octubre) }\end{array}$ & 33.0 & 13.0 & 14.0 & 60.0 \\
& & 19.0 & 7.0 & 9.0 & 35.0 \\
Concentración & noviembre & 1.3 & 1.1 & 0.8 & 0.8 \\
de fósforo soluble & diciembre & 0.2 & 0.3 & 0.3 & 0.6 \\
en ppm o g m & febrero & 0.2 & 0.1 & 0.2 & 0.4 \\
& septiembre & 1.6 & 1.4 & 0.9 & 0.3 \\
Caudales másicos & noviembre & 105.0 & 34.0 & 28.0 & 118.0 \\
eng s & diciembre & 16.0 & 9.0 & 11.0 & 88.0 \\
& febrero & 16.0 & 3.0 & 7.0 & 59.0 \\
& septiembre & 30.0 & 10.0 & 8.0 & 11.0 \\
\hline
\end{tabular}




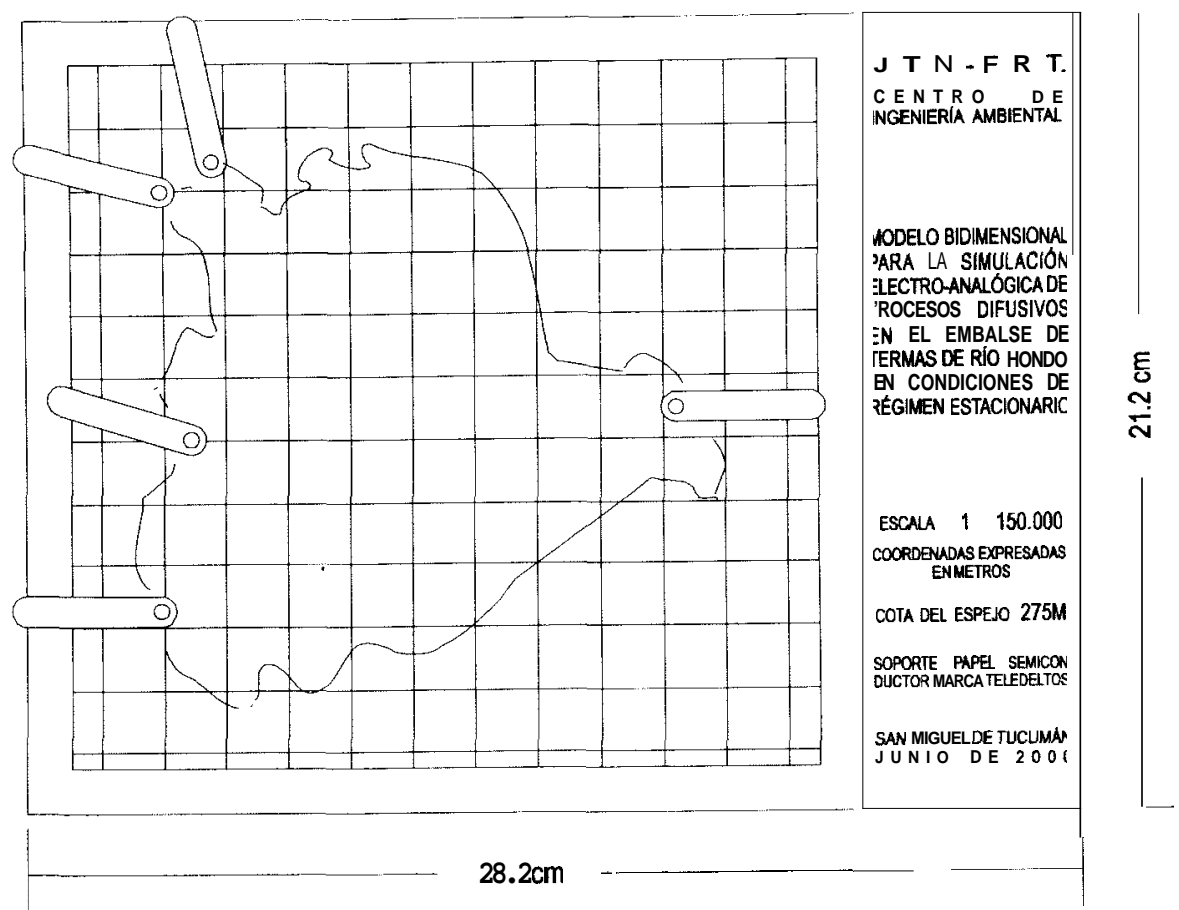

Figura 1. Esquema del Modelo Electro-analógico del Embalse de Termas de Rio Hondo Diagram of the Electro-analogue Model of the Termas de Rio Hondo Dam

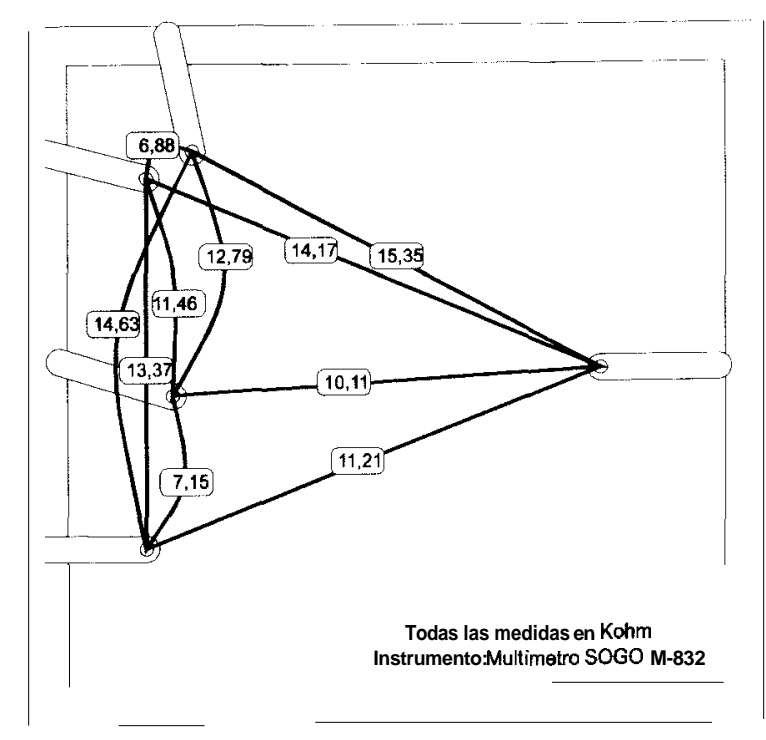

Figura 2. Circuito eléctrico equivalente del Modelo Analógico. Equivalent electric circuit of the Analogue Model.
El pantógrafo es un tablero rectangular plano, con un brazo articulado de dos piezas, montado en la esquina inferior derecha, como se ilustra en el Plano del Pantógrafo (Fig. 3). En cada articulación se ha instalado un potenciómetro y en el extremo distal del antebrazo se ha montado un electrodo sensor de bronce. Completa el conjunto, un pulsador que permite al usuario indicar al sistema de adquisición de datos, cuando el sensor se encuentra en el punto a medir. El Esquema Eléctrico del Pantógrafo (Fig. 4), muestra la disposición y características de los componentes del circuito de posicionamiento y censado de tensiones. Tanto el potenciómetro del hombro (PT1), como el del codo (PT2), se encuentran polarizados en sus extremos con $5 \mathrm{~V}$ y $0 \mathrm{~V}$, lo que permite al cursor generar una tensión variable dentro de este rango. Por ser potenciómetros lineales, la variación de tensión es lineal respecto del ángulo barrido o rotado por el brazo y el antebrazo. Se agregaron condensadores electrolíticos $(\mathrm{C} 1$ y $\mathrm{C} 2)$ a los cursores de los potenciómetros para dismi- 
nuir el ruido que capta el conversor analógicodigital (A / D) . El pulsador, normalmente abierto, permite a la entrada analógica IN3 mantener un nivel alto de tensión (aproximadamente $5 \mathrm{~V}$ ), a través de la resistencia R1 ; cuando se lo presiona, la tensión en dicha entrada es de $0 \mathrm{~V}$. La figura 5 , muestra el conexionado general.

Para la adquisición de datos y generación de las tensiones de control se dispuso de una interfase marca EDP 2 - Entorno de Desarrollo de Periféricos, de la firma $P C L A B$, Arg.. Este sistema permite la conexión de hasta ocho módulos de diverso tipo; en el presente trabajo, se emplearon dos analógicos y uno TTL. A este último se conectó una plaqueta que controla conversores A / D y D / A de 12 bits. La interfase que cuenta con drivers para su control desde aplicaciones MSWindows, dispone de ocho entradas analógicas multiplexadas con resolución de 8 bits; rango de 0 a $5 \mathrm{~V}$; una salida analógica con resolución de 8 bits; rango de 0 a $5 \mathrm{~V}$; conversor A / D , marca National ADC0804 (t $\mathrm{C}_{\mathrm{CONV}}: 80 \mu \mathrm{S}$ típico) y conversor D / A marca National DAC0800 (t) $\quad 10 \mu \mathrm{S}$ típico). El Módulo Analógico de 12 bits , tiene una entrada analógica con resolución de 12 bits ; rango: 0 a $10 \mathrm{~V}$; una salida analógica con resolución de 12 bits; rango de 0 a $10 \mathrm{~V}$; conversor A / D marca Maxim MAX574A $\left(\mathrm{t}_{\mathrm{CONV}}\right.$ : $25 \mu \mathrm{S}$ típico); conversor D / A marca National DAC1230 ( $\mathrm{t}_{\mathrm{CONV}}: 1 \mu \mathrm{S}$ típico).

En el circuito de interconexión, mostrado en el esquema de Conexionado General (Fig. 5 ), se utilizan tres salidas analógicas para polarizar tres de los cuatro electrodos del modelo electro-analógico. La primera y segunda salida utilizan conversores de 8 bits ; la tercera, un conversor de 12 bits. La señal medida se digitaliza en 8 bits .

\section{RESULTADOS}

Conjuntamente con la construcción y puesta a punto del modelo electro-analógico y del pantógrafo, se desarrolló en Visual Basic un programa de control para la captación, graficación y almacenamiento primario de los datos, el que consta de un Módulo Principal y de otro de Calibración;

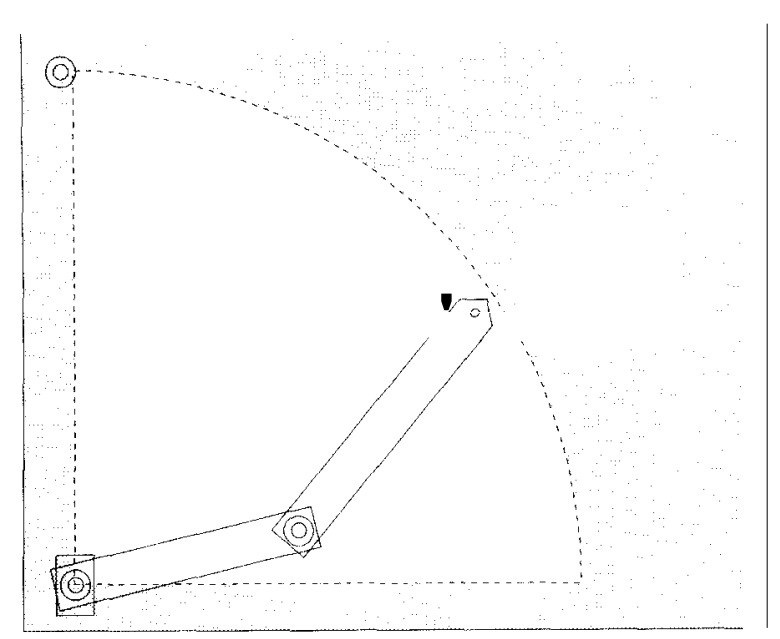

Figura 3. Plano del pantógrafo. Drawing of the pantograph

emplea para el control de las interfases analógicas una DLL (Dynamic Link Library) codificada en Borland C++ y cumple las siguientes funciones:

- Controlar la tensión aplicada en cada electrodo en función de los caudales másicos;

- Calcular la posición X - Y del electrodo sensor;

- Representar en pantalla en falso color, un mapa de contorno de tensión;

- Generar el archivo con las muestras u observaciones obtenidas.

El archivo de salida, tiene formato ASCII; cada línea corresponde a una terna $(x, y, v)$, perteneciente a la solución del problema. Las primeras componentes $(x, y)$, son longitudes enteras en milímetros, medidas desde el punto hacia el origen del sistema coordenado Gauss-Krüger, ubicado en el eje de rotación del potenciómetro del hombro del pantógrafo. La tercera componente es el valor de tensión medido ( $v$ ), convertido a unidades de caudal másico.

Con las corridas de simulación, realizadas conforme a los datos de la Tabla 1 sobre caudales hídricos; concentraciones y caudales másicos de fósforo soluble aportado por los tributarios del Embalse Frontal de Termas de Río Hondo y con- 
CONECTOR

$A E D P$
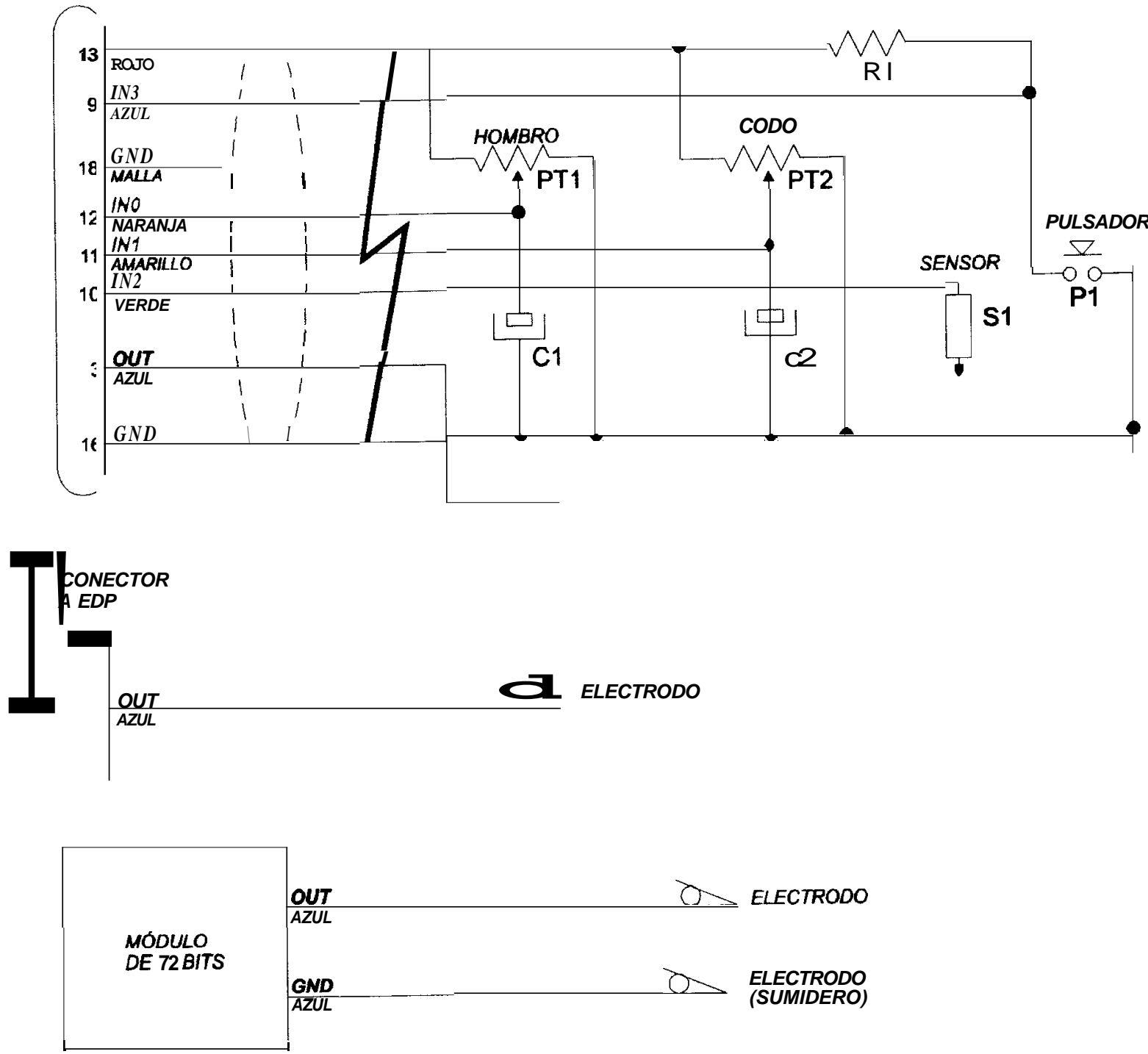

R1: resistencia 1,2Kohm 1/4W

C1, C2: condensador electrolitico 1uF / 50V

PT1, PT2: potenciómetro lineal 3,1 Kohm

$\mathrm{S} 1$ : electrodo de bronce

P1: pulsador NA

CN1: conector DB25 macho p/cable 


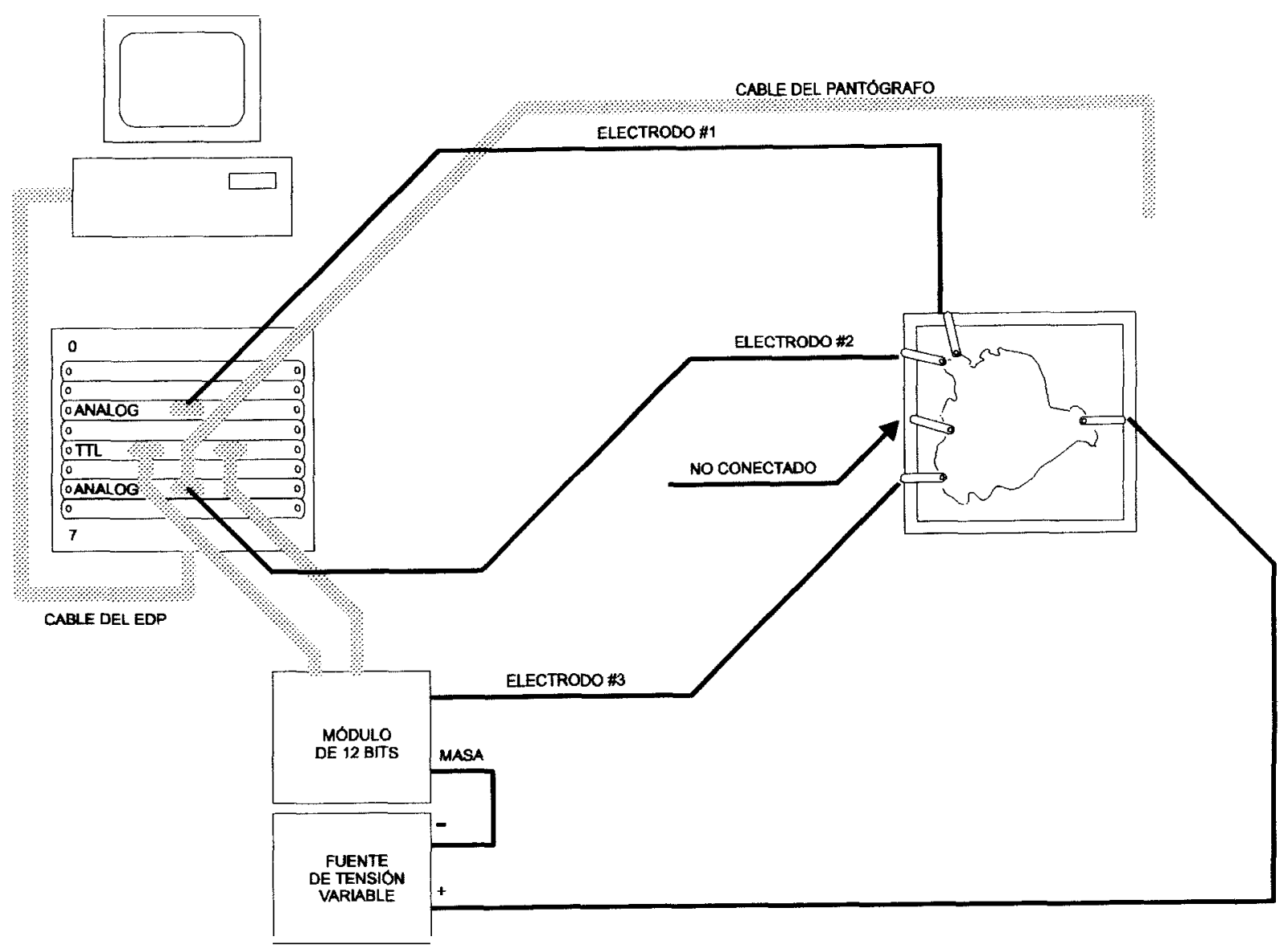

Figura 5. Conexionado general. General setup of electric connections.

siderando el espejo del Embalse en cota máxima, se obtienen archivos con extensión dat. Estos archivos son procesados con el programa Surfer 6.0, que genera archivos con extensión $\mathrm{grd}$. El logicial, ofrece opciones de graficación, tales como: líneas de contorno o curvas de nivel (Fig.6 y Fig. 8), o bien representaciones espaciales de superficies reticuladas; se tienen así, archivos gráficos con extensión plt, que pueden ser optimizados, resultando archivos con extensión opt (Fig. 7 y Fig. 9). En la figura 7 y en la figura 9, la superficie reticulada, es observada desde el cuadrante Suroeste.

\section{DISCUSIÓN Y CONCLUSIONES}

El modelo analógico-digital diseñado, construido y operado, genera isolíneas de potencial V o curvas equipotenciales que en condiciones de régimen estacionario, corresponden a la distribución de contaminantes y nutrientes, empleando la concentración $C$ como parámetro distribuido, pues en los procesos de difusión y mezclado, descriptos por la ecuación de Laplace, es posible emplear un sistema análogo de conducción eléctrica continua óhmica. Siendo el medio isótropo, con conductividad eléctrica $\sigma$, independiente de la posición $\mathbf{r}$, se 


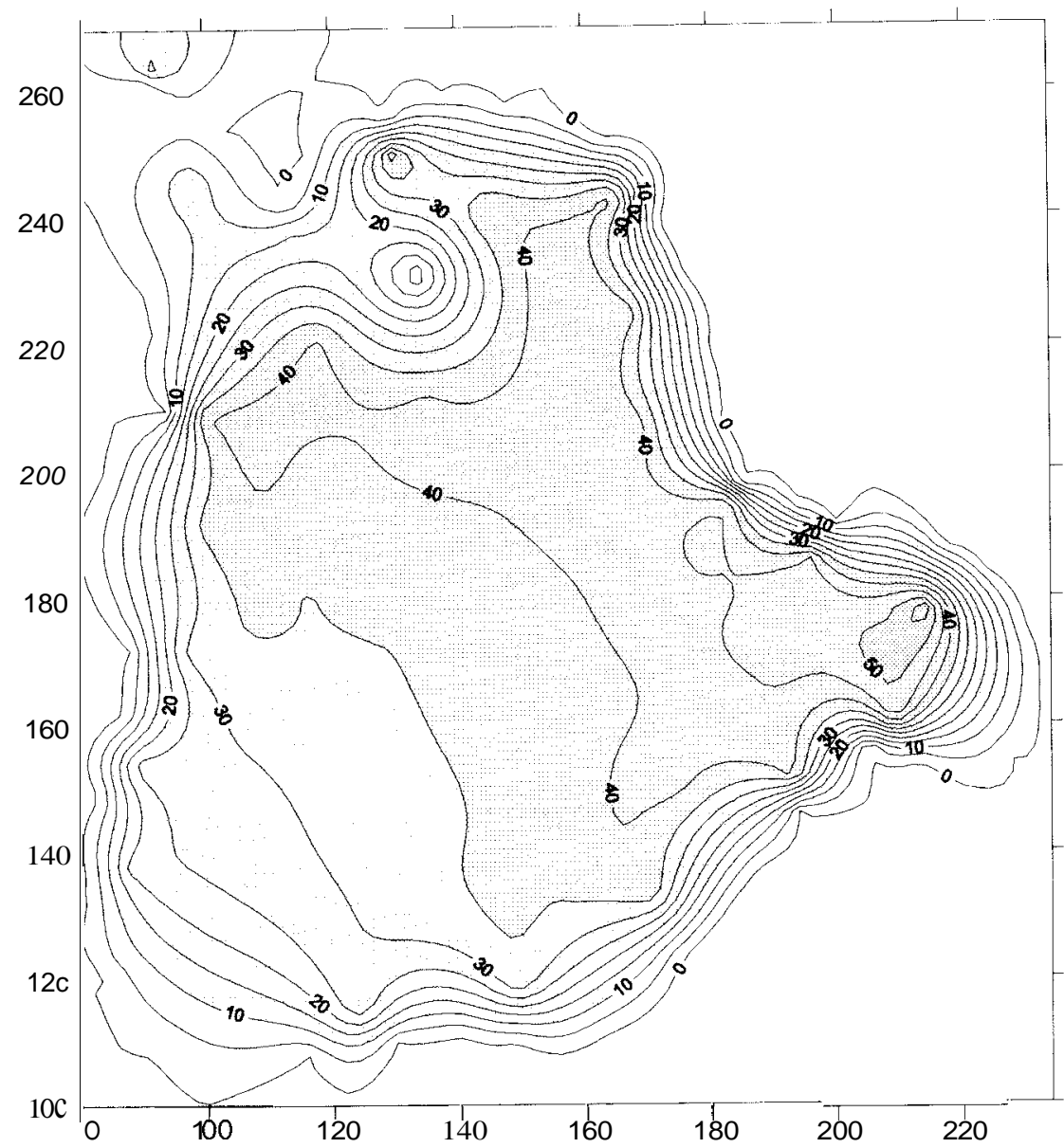

Figura 6. Curvas de igual concentración de ortofosfato soluble, en el Embalse Frontal de Termas de Río Hondo, correspondientes al mes de febrero. Isocurves of soluble orthophosphate concentration in the Termas de Río Hondo Frontal Darn, in February.

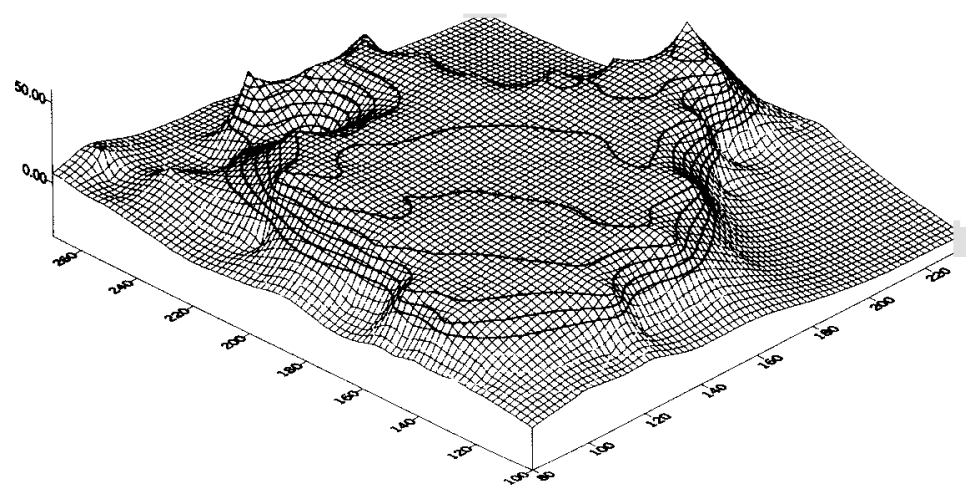

Figura 7. Superficie reticulada espacial de la variación de la concentración de ortofosfato soluble, en el Embalse Frontal de Termas de Río Hondo, correspondiente al mes de febrero. Grid showing ojthe spatial variation of the concentration of soluble orthophosphute, it7 the Termas de Río Hondo Frontal Dam. corresponding to the month in February. 


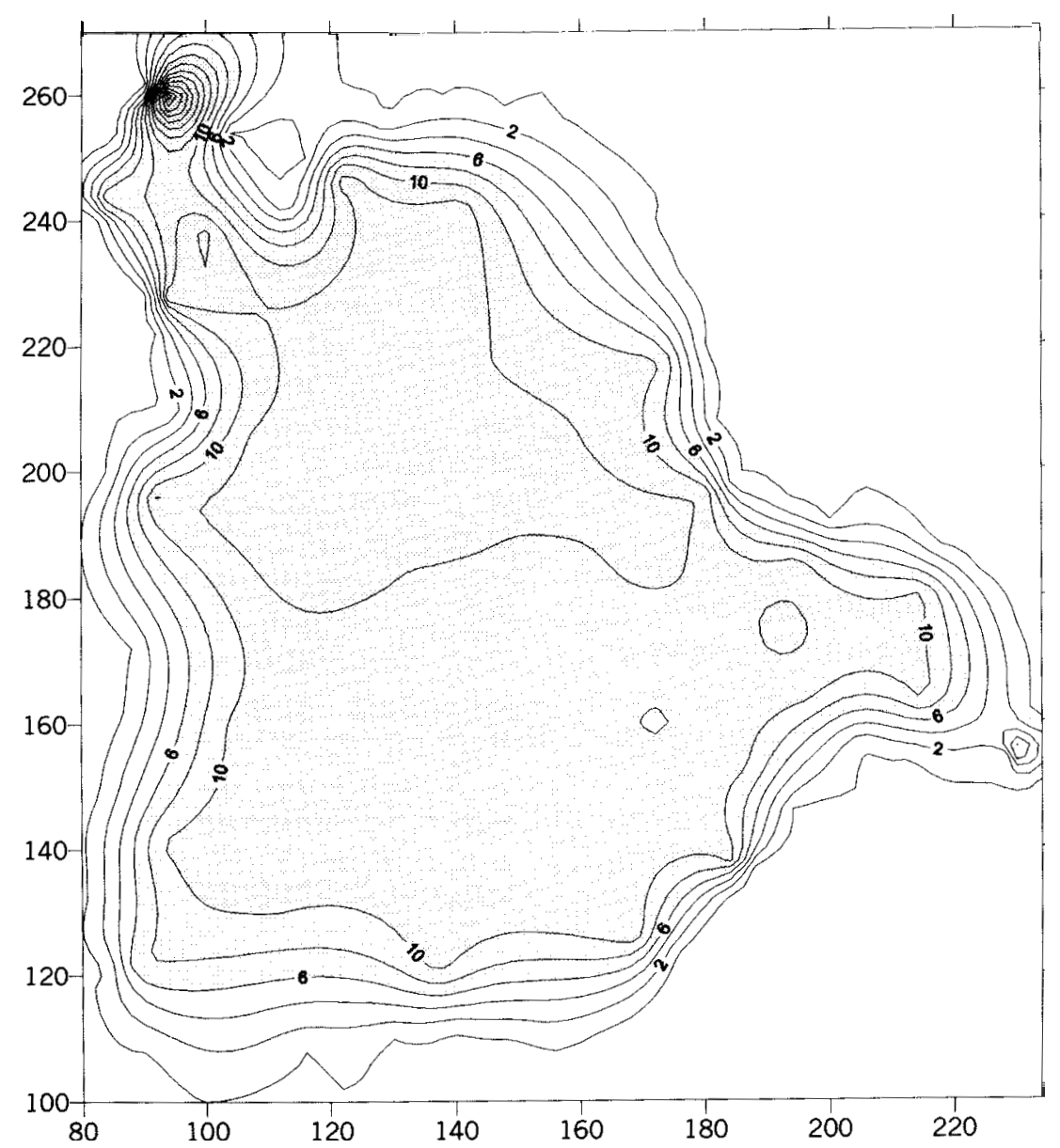

Figura 8. Curvas de igual concentración de ortofosfato soluble, en el Embalse Frontal de Termas de Río Hondo, correspondientes al mes de septiembre. Isocurves of soluble orthophosphate concentrution in the Termas de Rio Hondo Frontal Dam, in September:

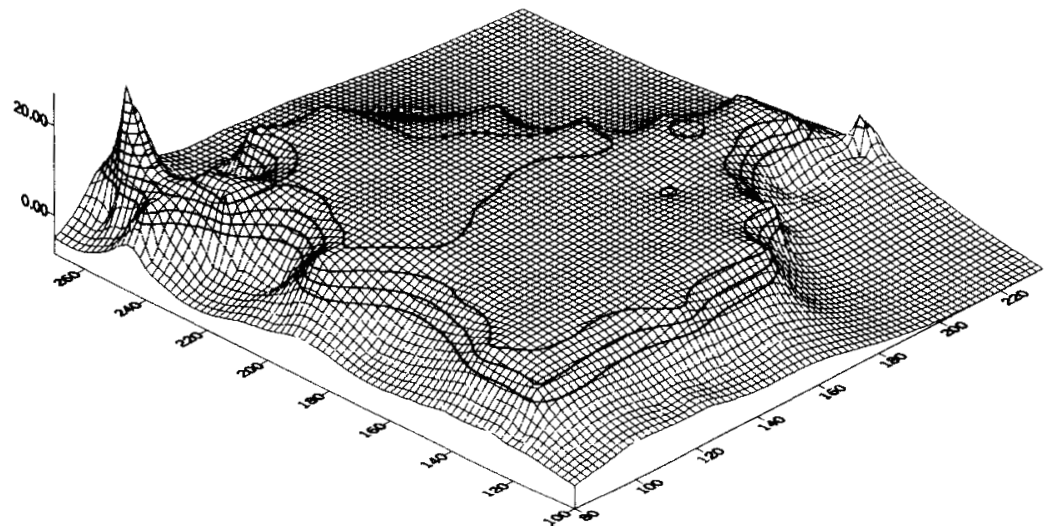

Figura 9. Superficie reticulada espacial de la variación de la concentración de ortofosfato soluble, en el Embalse Frontal de Termas de Río Hondo, correspondiente al mes de septiembre. Grid showing the spatial variation of the concentrution of soluble orthophosphate, in the Termas de Río Hondo Frontal Dam, corresponding to the month in September: 
tiene que: $\nabla^{2} \mathrm{~V}(\mathbf{r})=0$. Operando sobre un modelo bidimensional electro-analógico, se obtuvieron valores de tensión o potencial eléctrico. De este modo se generaron soluciones para la ecuación de Laplace $\nabla^{2} C(\mathbf{r})=0$, que describe la distribución espacial de la concentración $C$ de contaminantes y nutrientes, con coeficiente de difusión o mezclado $D$, independiente de la posición $\mathbf{r}$.

El procesamiento de los archivos de datos, conduce a la obtención de gráficos de líneas de contorno o curvas equipotenciales o bien a representaciones tridimensionales de superficies reticuladas. Cabe observar, que a pesar de ser nulos los valores de tensión fuera del área del modelo, el software de aplicación empleado, interpola entre el valor actual de tensión sobre el contorno del modelo y el marco del reticulado generado al superponer una grilla rectangular al espacio de trabajo; consiguientemente, la superficie objeto de estudio, se asimila al rectángulo que la inscribe.

La modelización se ha realizado en todos los casos, asumiendo que el espejo del embalse, se mantiene en la cota máxima; simplificación que no responde a la realidad, pues el nivel varía significativamente en el sistema real, conforme a las fluctuaciones estacionales del año hidrológico. Una mejora del planteo experimental, en este aspecto, conduciría a operar con contornos variables del papel semiconductor, en función de la cota del pelo libre.

\section{BIBLIOGRAFÍA}

AVRAMI, M. \& V. PASCHKIS. 1942.Application of an electric model to the study of two-dymensionai heat flow. Trans. Am. Inst. Chem. Engrs. 38: 631-634

CHOW, V.T., D.R. MAIDMENT \& L.W. MAYS. 1994. Hidrología Aplicada.; Mc Graw-Hill. Santafé de Bogotá. Colombia.
DAVIS, H.F. \& A.D. SNIDER. 1979. Introduction to VectorAnalysis. Fourth Edition. Allyn and Bacon, Inc. Boston Massachusetts, USA.

ERICKSON, P.A. 1994. A Practical Guide to Environmental Impact Assessment. Academic Press. San Diego, Ca (USA).

GLASSON, J., R. THERIVEL \& A. CHADWICK. 1994. Introduction to Environmental Impact Assessment: Principles and procedures, process, Practice and Prospects. The Natural and Built Environment Series, 1. UCL Press. London; Bristol, $\mathrm{Pa}$. UCL Press.

HOFFMANN, C., O. J. GRAIEB, J. G. PERERA, J. C. LUJÁN y R. CARABAJAL. 1991. Dispersión por cizallamiento en grandes masas de agua. VII Congreso Argentino de Fisicoquímica (Córdoba, Argentina, 22-25/04/91). Tucumán (Argentina). Universidad Tecnológica Nacional. 9 pp.

HOFFMANN, C., O. J. GRAIEB, J. G. PERERA, J. C. LUJÁN y R. CARABAJAL. 1993. Sobre el modelo de dos capas para describir la dispersión por deslizamiento en lagos. VIII Congreso Argentino de Fisicoquímica (Mar del Plata, Argentina, 19-22/04/93). Tucumán (Argentina). Universidad Tecnológica Nacional. 7 pp.

LIEBMANN, G. 1953. Electric Analogues. Brit. J. Appl. Phys., 4: 193-199

PERERA, J. G., O. J. GRAIEB, J. C. LUJÁN, J. S. PAGANI y A. E. ORTEGA. 1994. Modelo matemático del proceso de colmatación del embalse de Termas de Río Hondo, basado en el empleo de un modelo reducido hidráulico. Tucumán (Argentina). Universidad Tecnológica Nacional. Memoria de un Proyecto de Investigación. 147 pp.

PRASUHN, A. L.. 1987. Fundamentals of Hydraulic Engineering. Holt, Rinehart and Winston International Edition. NY (USA).

WANIELISTA, M. P.. 1990. Hydrology and Water Quantity Control. John Wiley. 